\title{
J. MORGAN-SCIARRINO
}

\section{Algorithme d'approximation interne de problèmes de point de selle avec contraintes via l'optimisation}

RAIRO - Analyse numérique, tome 14, no 2 (1980), p. 189-202.

$<$ http://www.numdam.org/item?id=M2AN_1980_14_2_189_0>

C AFCET, 1980, tous droits réservés.

L'accès aux archives de la revue «RAIRO - Analyse numérique » implique l'accord avec les conditions générales d'utilisation (http://www.numdam.org/ legal.php). Toute utilisation commerciale ou impression systématique est constitutive d'une infraction pénale. Toute copie ou impression de ce fichier doit contenir la présente mention de copyright.

\section{Numdam}




\title{
ALGORITHME D'APPROXIMATION INTERNE DE PROBLĖMES DE POINT DE SELLE AVEC CONTRAINTES VIA L'OPTIMISATION $\left({ }^{*}\right)$
}

\author{
par J Morgan-SCIARrino ( ${ }^{\mathbf{1}}$ ) \\ Communique par J CEA
}

\begin{abstract}
Resume - On presente dans cet article un procede d'approximation interne d'un probleme de point de selle avec contraintes non limitees par une suite de problemes d'optımisation pouvant être consideres comme sans contraintes Cecl conduit a un algorithme dont chaque iteration consiste en une maximisation par rapport a l'une des variables et une minimisation par rapport a l'autre On en demontre la convergence forte dans des espaces de Hilbert et on donne des majorations d'erreurs
\end{abstract}

Abstract - A saddle-point problem with several unbounded constraints is transformed by means of an internal penalty function into the solution of a sequence of unconstrained optimization problems This leads to an iterative implementable procedure each iteration consists of two steps of optimization mmmm_ation in one variable and maximization on the other not on finding a saddle-point of the corresponding penalty saddl function This method is shoun to be strongly convergent in Hilbert spaces and can proude an crimulk of arm,

\section{INTRODUCTION}

Solt $K_{1}$ (respectivement $K_{2}$ ) un sous-ensemble convexe ferme d'un espace de Hilbert réel $V_{1}\left(\right.$ resp $V_{2}$ ) et une fonctionnelle réelle $J$ definie sur $K_{1} \times K_{2}$

On considère le probleme suivant

Probleme $(P)$ trouver un point de selle de $J$ sur $K_{1} \times K_{2}$, c'est-à-dire un couple $\left(u_{1}, u_{2}\right) \in K_{1} \times K_{2}$ tel que

$$
J\left(u_{1} v_{2}\right) \leqq J\left(u_{1}, u_{2}\right) \leqq J\left(v_{1}, u_{2}\right), \quad \forall\left(v_{1}, v_{2}\right) \in K_{1} \times K_{2}
$$

Diverses methodes d'approxımation de problemes d'optımısation ont été adaptées a l'approxımation de problèmes de point de selle avec contraintes [1] ma1s ces méthodes ne peuvent être employees que dans le cas où les fonctionnelles introduites par le probleme sont partout definies L'adaptation de

(*) Reçu octobre 1978

( ${ }^{1}$ Istituto dı Matematıca dell Universita dı Napolı Italıe

R A I R O Analyse numerique/Numerical Analysıs, 0399-0516/1980/189/\$ 500

(C) AFCET-Bordas-Dunod 
la méthode de A. V. Fiacco et G. P. McCormick aux problèmes de point de selle avec contraintes $[8,6]$ a permis d'établir une méthode d'approximation interne qui ne nécessite pas que les fonctionnelles soient partout définies. Toutefois les résultats établis ne font que transformer le problème de point de selle avec contraintes en un problème de point de selle pouvant être considéré comme sans contraintes et ne fournissent donc pas de moyens simples pour approcher numériquement le point de selle cherché. Le but de cet article est de présenter un procédé d'approximation interne du problème $(\mathrm{P})$ par une suite de problèmes d'optimisation pouvant être considérés comme sans contraintes ou plus précisément un algorithme dont chaque itération consiste en la résolution de deux problèmes d'optimisation pouvant être considérés comme sans contraintes, maximisation par rapport à l'une des variables puis minimisation par rapport à l'autre. Ceci permettra, après discrétisation, d'utiliser d'une part les résultats établis par A. V. Fiacco et G. P. McCormick en optimisation et d'autre part des techniques bien connues d'optimisation comme celle très simple d'emploi des variations locales [2].

\section{HYPOTHẼSES}

Pour $i=1,2$ on note $(,)_{i}$ le produit scalaire dans $V_{i},\|\| \|_{i}$ la norme sur $V_{i}$ et $\langle,\rangle_{i}$ la dualité entre l'espace $V_{i}$ et son dual $V_{i}^{\prime}$.

$1^{\circ}$ La fonctionnelle $J$, définie sur $K_{1} \times K_{2}$, vérifie :

$J$ est deux fois continûment différentiable sur $K_{1} \times K_{2}$ et pour $i=1,2$ les opérateurs linéaires $D_{i}^{2} J\left(v_{1}, v_{2}\right): V_{i} \rightarrow V_{i}^{\prime}$ sont surjectifs pour tout couple $\left(v_{1}, v_{2}\right)$ de $K_{1} \times K_{2}$.

$J$ est fortement convexe-concave sur $K_{1} \times K_{2}$ c'est-à-dire : il existe deux constantes positives $T_{1}$ et $T_{2}$ telles que

$$
\left.\begin{array}{c}
J\left(v_{1}+w_{1}, v_{2}\right)-J\left(v_{1}, v_{2}\right) \geqq\left\langle D_{1} J\left(v_{1}, v_{2}\right), w_{1}\right\rangle_{1}+T_{1}\left\|w_{1}\right\|_{1}^{2} \\
J\left(v_{1}, v_{2}+w_{2}\right)-J\left(v_{1}, v_{2}\right) \leqq\left\langle D_{2} J\left(v_{1}, v_{2}\right), w_{2}\right\rangle_{2}-T_{2}\left\|w_{2}\right\|_{2}^{2} \\
\forall\left(v_{1}, v_{2}\right) \in K_{1} \times K_{2}, \quad \forall\left(w_{1}, w_{2}\right) \in K_{1} \times K_{2} .
\end{array}\right\}
$$

Pour tout couple $\left(v_{1}, v_{2}\right)$ de $K_{1} \times K_{2}$ l'application $D_{2} D_{1} J\left(v_{1}, v_{2}\right): V_{2} \rightarrow V_{1}^{\prime}$ est indépendante de l'une des deux variables. Par la suite on supposera que l'on se trouve dans le cas où cette application est indépendante de la variable $v_{1}$.

Il existe $k$, réel positif, tel qu'on ait :

$$
\left.\begin{array}{c}
\left\|\left[D_{1}^{2} J\left(v_{1}, v_{2}\right)\right]^{-1}\right\| \cdot\left\|D_{2} D_{1} J\left(v_{1}, v_{2}\right)\right\|^{2}\left\|\left[D_{2}^{2} J\left(v_{1}, v_{2}\right)\right]^{-1}\right\| \leqq k \\
\forall\left(v_{1}, v_{2}\right) \in K_{1} \times K_{2}
\end{array}\right\}
$$

R.A.I.R.O. Analyse numérique/Numerical Analysis 
$2^{\circ}$ Pour $i=1,2$ on considère des fonctionnelles $G_{i, j}\left(j=1, \ldots, q_{i}\right)$ vérifiant : $G_{i, j}$ est concave et deux fois continûment différentiable sur l'ensemble $K_{i}$.

On suppose alors l'ensemble $K_{i}$ défini de la manière suivante :

$$
K_{i}=\left\{v_{i} \in V_{i} / G_{i, j}\left(v_{\imath}\right) \geqq 0, j=1, \ldots, q_{i}\right\}
$$

et tel que

$$
\stackrel{\circ}{K}_{i}=\left\{v_{i} \in V_{\imath} / G_{i, j}\left(v_{i}\right)>0, j=1, \ldots, q_{i}\right\}
$$

est un ensemble non vide.

Remarque 2.1 : Les hypothèses (2.1) et (2.2) impliquent que, pour $i=1$, 2 , et pour tout couple $\left(v_{1}, v_{2}\right)$ de $K_{1} \times K_{2}$, les opérateurs $D_{i}^{2} J\left(v_{1}, v_{2}\right)$ sont des homéomorphismes linéaires de $V_{i}$ sur $V_{i}^{\prime}$.

REMARQUe 2.2 : L'hypothèse (2.3) est notamment vérifiée dans le cas suivant :

$$
J\left(v_{1}, v_{2}\right)=J_{1}\left(v_{1}\right)+J_{2}\left(v_{2}\right)+b\left(v_{2}, v_{1}\right),
$$

où $J_{1}$ (respectivement $J_{2}$ ) est une fonctionnelle deux fois continûment différentiable sur $K_{1}$ (resp. $K_{2}$ ) et $b$ est une fonctionnelle linéaire continue par rapport à l'une des variables et continûment différentiable par rapport à l'autre.

REMARQUE 2.3 : On considère le cas d'une fonctionnelle de type quadratique, c'est-à-dire

$$
J\left(v_{1}, v_{2}\right)=a_{1}\left(v_{1}, v_{1}\right)-a_{2}\left(v_{2}, v_{2}\right)+b\left(v_{2}, v_{1}\right)-L_{1}\left(v_{1}\right)+L_{2}\left(v_{2}\right),
$$

où $a_{1}$ et $a_{2}$ sont des formes bilinéaires symétriques et coercives, $b$ est une forme bilinéaire continue et $L_{1}$ et $L_{2}$ sont des formes linéaires continues. Ce cas est donc un cas particulier de celui défini dans la remarque 2.2.

Si les opérateurs $A_{1}$ et $A_{2}$ associés aux formes bilinéaires $a_{1}$ et $a_{2}$ sont inversibles alors les hypothèses (2.1), (2.2), (2.3) et (2.4) sont vérifiées et de plus on peut prendre

$$
k=\frac{1}{4}\left\|A_{1}^{-1}\right\| \cdot\|B\|^{2}\left\|A_{2}^{-1}\right\|,
$$

où $B$ est l'opérateur associé à la forme bilinéaire $b$.

\section{DESCRIPTION DU PROCÉDÉ D'APPROXIMATION}

$1^{\circ}$ Rappelons d'abord brièvement certains résultats, démontrés dans [8], que nous utiliserons par la suite. 
Pour $i=1,2$ on se donne une suite de nombres positifs $r_{i}$ et une fonction positive croissante $\varepsilon_{i}: r_{i} \rightarrow \varepsilon_{i}\left(r_{i}\right)$ telle que $\varepsilon_{i}\left(r_{i}\right) \rightarrow 0$ quand $r_{i} \rightarrow 0$ et on pose $r-(r, r, r, \mathcal{G})$ définit une fonctionnelle $J_{r}$ sur $\hat{K}_{1} \times \hat{K}_{2}$ de la manière suivante : surtante :

$$
J_{r}\left(v_{1}, v_{2}\right)=J\left(v_{1}, v_{2}\right)+\varepsilon_{1}\left(r_{1}\right)_{1}^{q_{1}} \frac{1}{G_{1, j}\left(v_{1}\right)}-\varepsilon_{2}\left(r_{2}\right) \sum_{1}^{q_{2}} \frac{1}{G_{2, j}\left(v_{2}\right)}
$$

et on introduit deux applications univoques $\Phi_{r}: \stackrel{\circ}{K}_{2} \rightarrow \check{K}_{1}$ et $\alpha_{r}: \check{K}_{1} \rightarrow \check{K}_{2}$ définies par

$$
\begin{aligned}
& J_{r}\left(\Phi_{r}\left(v_{2}\right), v_{2}\right)=\inf _{v_{1} \in \dot{K}_{1}} J_{r}\left(v_{1}, v_{2}\right), \\
& J_{r}\left(v_{1}, \alpha_{r}\left(v_{1}\right)\right)=\sup _{v_{2} \in \dot{K}_{2}} J_{r}\left(v_{1}, v_{2}\right) .
\end{aligned}
$$

On considère alors le problème approché suivant :

Problème $\left(\mathrm{P}_{r}\right)$ : trouver un point de selle de $J_{r}$ sur $K_{1}^{\circ} \times \check{K}_{2}$, c'est-à-dire un couple $\left(u_{1, r}, u_{2, r}\right) \in \mathscr{K}_{1} \times \check{K}_{2}^{\circ}$ tel que :

$$
\left.\begin{array}{c}
J_{r}\left(u_{1, r}, v_{2}\right) \leqq J_{r}\left(u_{1, r}, u_{2, r}\right) \leqq J_{r}\left(v_{1}, u_{2, r}\right) \\
\forall\left(v_{1}, v_{2}\right) \in \dot{K}_{1} \times K_{2} .
\end{array}\right\}
$$

On démontre l'existence et l'unicité de sa solution et la convergence forte de cette solution vers la solution du problème ( $(\mathfrak{)})$. De plus $u_{1, r}$ et $u_{2, r}$ vérifient :

$$
\begin{gathered}
u_{1, r}=\Phi_{r}\left(u_{2, r}\right) \quad \text { et } \quad u_{2, r}=\alpha_{r}\left(u_{1, r}\right), \\
\left\|u_{i, r}-u_{i}\right\|^{2} \leqq \frac{1}{T_{i}}\left[\varepsilon_{1}\left(r_{1}\right) \sum_{1}^{q_{1}} \frac{1}{G_{1, j}\left(u_{1, r}\right)}+\varepsilon_{2}\left(r_{2}\right) \sum_{1}^{q_{2}} \frac{1}{G_{2, j}\left(u_{2, r}\right)}\right] .
\end{gathered}
$$

$2^{\circ}$ Dans cet article on introduit une nouvelle application univoque $T_{r, \beta}$ : $\check{K}_{1} \rightarrow \check{K}_{1}$ définie par

$$
T_{r, \beta}=(1-\beta)\left(\Phi_{r} \circ \alpha_{r}\right)+\beta I_{V_{1}}, \quad \beta \in \mathbb{R}
$$

et on montre que pour certaines valeurs de $\beta$ cette application est contractante (de rapport de contraction indépendant de $r$ ) ce qui permet de construire un procédé itératif de la manière suivante : on se donne une double suite $r_{n}=\left(r_{1, n}\right.$, $r_{2, n}$ ) de nombres positifs décroissants vers zéro quand $n \rightarrow+\infty$ et une valeur arbitraire $x_{1,0}$ de $K_{1}^{\circ}$, on donne au paramètre $\beta$ la valeur $\beta_{0}$ qui rend le rapport de contraction minimal et on définit une double suite $\left(x_{1, n}, x_{2, n}\right)$ à partir de $x_{1,0}$ par :

$$
\begin{gathered}
x_{2, n}=\alpha_{r_{n}}\left(x_{1, n-1}\right), \\
x_{1, n}=\left(1-\beta_{0}\right) \Phi_{r_{n}}\left(x_{2, n}\right)+\beta_{0} x_{1, n-1}=T_{r_{n}, \beta_{0}}\left(x_{1, n-1}\right) .
\end{gathered}
$$


On démontre alors que la double suite $\left(x_{1, n}, x_{2, n}\right)$ converge fortement vers la solution $\left(u_{1}, u_{2}\right)$ du problème $(\mathrm{P})$ et on établit des majorations d'erreurs.

Remarque 3.1: A chaque itération on détermine $x_{2, n}$ en maximisant $J_{r_{n}}$ par rapport à une variable et $\Phi_{r_{n}}\left(x_{2, n}\right)$ en minimisant par rapport à l'autre. La détermination de $x_{1, n}$ et $x_{2, n}$ conduit donc à la résolution de deux problèmes d'optimisation.

Remarque $3.2:$ Si dans l'hypothèse (2.3) on suppose que l'application $D_{2} D_{1}$ $J\left(v_{1}, v_{2}\right)$ de $V_{2}$ dans $V_{1}^{\prime}$ est indépendante de $v_{2}$ (au lieu de l'être de $v_{1}$ ) on peut remplacer l'algorithme défini par (3.7) et (3.8) par un algorithme analogue faisant intervenir l'application $\alpha_{r} \circ \Phi_{r}$ au lieu de l'application $\Phi_{r} \circ \alpha_{r}$ :

$$
\begin{gathered}
x_{1, n}=\Phi_{r_{n}}\left(x_{2, n-1}\right), \\
x_{2, n}=\left(1-\beta_{0}\right) \alpha_{r_{n}}\left(x_{1, n}\right)+\beta_{0} x_{2, n-1} .
\end{gathered}
$$

Remarque 3.3 : Pour déterminer un point de départ du procédé, c'est-à-dire un élément $x_{1,0}$ de $\check{K}_{1}$ on peut utiliser la méthode indiquée par A. V. Fiacco dans [3], après discrétisation.

La démonstration de la convergence du procédé exige la connaissance de résultats préliminaires que nous présentons sous forme de propositions dans le paragraphe suivant.

\section{PÉNALISATION INTERNE ET OPTIMISATION}

Étudions les propriétés de l'application $\Phi_{r} \circ \alpha_{r}: \AA_{1} \rightarrow K_{1}$.

PRoposition 4.1. - L'application $\Phi_{r} \circ \alpha_{r}$ (respectivement $\left.\alpha_{r} \circ \Phi_{r}\right)$ admet l'élément $u_{1, r}\left(\right.$ resp. $\left.u_{2, r}\right)$, solution du problème $\left(\mathrm{P}_{r}\right)$, comme unique point fixe sur $K_{1}\left(\right.$ resp. $\left.\stackrel{K}{K}_{2}\right)$ pour tout $r$ positif.

Démonstration: D'après (3.5) on a

$$
\left(\Phi_{r} \circ \alpha_{r}\right)\left(u_{1, r}\right)=u_{1, r} \quad \text { et } \quad\left(\alpha_{r} \circ \Phi_{r}\right)\left(u_{2, r}\right)=u_{2, r},
$$

par suite $u_{1, r}$ (resp. $u_{2, r}$ ) est point fixe de $\Phi_{r} \circ \alpha_{r}$ (resp. de $\alpha_{r} \circ \Phi_{r}$ ). Montrons l'unicité de ce point fixe. Supposons pour cela qu'il existe un autre point fixe $\bar{u}_{1, r}$ $\operatorname{de} \Phi_{r} \circ \alpha_{r}$ sur $K_{1}$, distinct de $u_{1, r}$. Posons $\bar{u}_{2, r}=\alpha_{r}\left(\bar{u}_{1, r}\right)$. On a $\Phi_{r}\left(\bar{u}_{2, r}\right)=\bar{u}_{1, r}$. Par suite

$$
J_{r}\left(\bar{u}_{1, r}, \bar{u}_{2, r}\right)=\inf _{\imath_{1} \in \hat{K}_{1}} J_{r}\left(v_{1}, \bar{u}_{2, r}\right)=\sup _{v_{2} \in \hat{K}_{2}} J_{r}\left(\bar{u}_{1, r}, v_{2}\right)
$$

et $\left(\bar{u}_{1, r}, \bar{u}_{2, r}\right)$, est donc un point de selle de $J_{r}$ sur $\dot{K}_{1} \times \dot{K}_{2}$. Le point de selle de $J_{r}$ sur $\hat{K}_{1} \times K_{2}$ étant unique on en déduit le résultat énoncé. 
Proposition $4.2:$ L'application $\left(-\Phi_{r} \circ \alpha_{r}\right): \AA_{1} \rightarrow \AA_{1}$ est différentiable et monotone pour tout $r$ positif, c'est-à-dire : pour tout couple

$$
\left(v_{1}, w_{1}\right) \in \check{K}_{1} \times K_{1}\left(\left(\Phi_{r} \circ \alpha_{r}\right)\left(v_{1}\right)-\left(\Phi_{r} \circ \alpha_{r}\right)\left(w_{1}\right), v_{1}-w_{1}\right)_{1} \leqq 0 .
$$

Démonstration : En utılisant le fait que les éléments $\Phi_{r}\left(v_{2}\right)$ et $\alpha_{r}\left(v_{1}\right)$ vérifient :

$$
D_{1} J_{r}\left(\Phi_{r}\left(v_{2}\right), v_{2}\right)=0 \quad \text { et } \quad D_{2} J_{r}\left(v_{1}, \alpha_{r}\left(v_{1}\right)\right)=0
$$

et en appliquant le théorème des fonctions implicites on montre que $\Phi_{r} \circ \alpha_{r}$ est différentiable sur $\check{K}_{1}$ et de plus, pour tout $v_{1} \in K_{1}^{\circ}$ on a

$$
\begin{aligned}
& {\left[D\left(\Phi_{r} \circ \alpha_{r}\right)\right]\left(v_{1}\right)=\left[D_{1}^{2} J_{r}\left(\left(\Phi_{r} \circ \alpha_{r}\right)\left(v_{1}\right), \alpha_{r}\left(v_{1}\right)\right)\right]^{-1} } \\
& \circ\left[D_{2} D_{1} J_{r}\left(\left(\Phi_{r} \circ \alpha_{r}\right)\left(v_{1}\right), \alpha_{r}\left(v_{1}\right)\right)\right] \circ\left[D_{2}^{2} J_{r}\left(v_{1}, \alpha_{r}\left(v_{1}\right)\right)\right]^{-1} \\
& \\
& \quad \circ\left[D_{1} D_{2} J_{r}\left(v_{1}, \alpha_{r}\left(v_{1}\right)\right)\right] .
\end{aligned}
$$

Démontrons maintenant la monotonie.

En applıquant les règles de dérivation à la fonctionnelle $J_{r}$ définie par (3.1) on obtient :

$$
\begin{gathered}
D_{1}^{2} J_{r}\left(v_{1}, v_{2}\right)=D_{1}^{2} J\left(v_{1}, v_{2}\right)+\varepsilon_{1}\left(r_{1}\right) \sum_{1}^{q_{1}} D^{2}\left(\frac{1}{G_{1, J}}\right)\left(v_{1}\right), \\
D_{2}^{2} J_{r}\left(v_{1}, v_{2}\right)=D_{2}^{2} J\left(v_{1}, v_{2}\right)-\varepsilon_{2}\left(r_{2}\right) \sum_{1}^{q_{2}} D^{2}\left(\frac{1}{G_{2, J}}\right)\left(v_{2}\right), \\
D_{1} D_{2} J_{r}\left(v_{1}, v_{2}\right)=D_{1} D_{2} J\left(v_{1}, v_{2}\right) .
\end{gathered}
$$

Pour $\imath=1,2$ appelons $U_{\imath}$ l'isomorphisme canonıque de $V_{\imath}$ sur $V_{\imath}^{\prime}$ définı par

$$
\left(v_{l}, w_{l}\right)_{l}=U_{l}\left(w_{\imath}\right) \cdot v_{l}=\left\langle U_{l}\left(w_{\imath}\right), v_{l}\right\rangle_{l} .
$$

Soit $V$ un espace de Hilbert. On rappelle qu'un opérateur symétrique de $\mathscr{L}(V, V)$ est dit positif si $(A v, v)_{V} \geqq 0$ pour tout $v \in V$ et négatıf si $(A v, v)_{V} \leqq 0$ pour tout $v \in V$.

D'après les hypothèses (2.1) et (2.2) l'opérateur $U_{1}^{-1} \mathrm{o}_{1}^{2} J\left(v_{1}, v_{2}\right)$ de $\mathscr{L}\left(V_{1}, V_{1}\right)$ [resp. l'opérateur $U_{2}^{-1} \circ D_{2}^{2} J\left(v_{1}, v_{2}\right)$ de $\mathscr{L}\left(V_{2}, V_{2}\right)$ ] est donc positif (resp. négatif) pour tout couple $\left(v_{1}, v_{2}\right) \in K_{1} \times K_{2}$. De plus on sait que s1 $A$ est un opérateur positıf de $\mathscr{L}(V, V)$ alors l'opérateur $A+\lambda I_{V}$ est positıf inversible et tel que

$$
\left\|\left(A+\lambda I_{V}\right)^{-1}\right\|_{\mathscr{L}(V V)}^{-1} \geqq \lambda \quad[9] \quad \text { pour } \quad \lambda>0 .
$$


On peut donc en déduire que pour tout couple $\left(v_{1}, v_{2}\right)$ de $\AA^{\circ} \times \check{K}_{2}$ l'opérateur $U_{1}^{-1} \circ D_{1}^{2} J_{r}\left(v_{1}, v_{2}\right)$ de $\mathscr{L}\left(V_{1}, V_{1}\right)$ [resp. l'opérateur $U_{2}^{-1} \circ D_{2}^{2} J_{r}\left(v_{1}, v_{2}\right)$ de $\left.\mathscr{L}\left(V_{2}, V_{2}\right)\right]$ est positif et inversible (resp. négatif et inversible) et de plus

$$
\begin{aligned}
&\left\|\left[D_{1}^{2} J_{r}\left(v_{1}, v_{2}\right)\right]^{-1}\right\|_{\mathscr{L}\left(V_{1}^{\prime} V_{1}\right)} \leqq\left\|\left[D_{1}^{2} J\left(v_{1}, v_{2}\right)\right]^{-1}\right\|_{\mathscr{L}\left(V_{1}^{\prime}, V_{1}\right)}, \\
&\left\|\left[D_{2}^{2} J_{r}\left(v_{1}, v_{2}\right)\right]^{-1}\right\|_{\mathscr{L}\left(V_{2}^{\prime}, V_{2}\right)} \leqq\left\|\left[D_{2}^{2} J\left(v_{1}, v_{2}\right)\right]^{-1}\right\|_{\mathscr{L}\left(V_{2}^{\prime}, V_{2}\right)} .
\end{aligned}
$$

Dans le but d'achever la démonstration de la proposition démontrons maintenant le lemme suivant :

Lemme 4.1: Si l'homéomorphisme linéaire $A_{1}$ de $V_{1}$ sur $V_{1}^{\prime}$ (resp. l'homéomorphisme linéaire $A_{2}$ de $V_{2}$ sur $V_{2}^{\prime}$ ) est tel que l'opérateur $U_{1}^{-1} \circ A_{1}$ de $\mathscr{L}\left(V_{1}, V_{1}\right)$ [resp. l'opérateur $U_{2}^{-1} \circ A_{2}$ de $\left.\mathscr{L}\left(V_{2}, V_{2}\right)\right]$ est symétrique positif (resp. symétrique négatif) et si l'application $B$ appartient à $\mathscr{L}\left(V_{1}, V_{2}^{\prime}\right)$ alors l'opérateur $A_{1}^{-1} \circ^{t} B \circ A_{2}^{-1} \circ B$ de $\mathscr{L}\left(V_{1}, V_{1}\right)$ (ou ${ }^{t} B$ est l'application transposée de $B)$ est symétrique négatif.

Démonstration: En effet l'opérateur $A_{2}^{-1} \mathrm{o} U_{2}$ de $\mathscr{L}\left(V_{2}, V_{2}\right)$ [resp. l'opérateur $C_{1}=A_{1}^{-1} \circ U_{1}$ de $\left.\mathscr{L}\left(V_{1}, V_{1}\right)\right]$ étant symétrique négatif(resp. symétrique positif) on peut vérifier que l'opérateur $C_{2}=U_{1}^{-1}{ }^{t}{ }^{t} \circ A_{2}^{-1} \circ B$ est symétrique négatif et que les opérateurs $C_{1}$ et $C_{2}$ commutent. Le produit $A_{1}^{-1} \mathrm{o}^{t} B \circ A_{2}^{-1} \circ B$ des opérateurs $C_{1}$ et $C_{2}$ est donc un opérateur symétrique négatif de $\mathscr{L}\left(V_{1}, V_{1}\right)$.

Pour utiliser ce lemme on pose

$$
A_{1}=D_{1}^{2} J_{r}\left(\left(\Phi_{r} \circ \alpha_{r}\right)\left(v_{1}\right), \alpha_{r}\left(v_{1}\right)\right), \quad A_{2}=D_{2}^{2} J_{r}\left(v_{1}, \alpha_{r}\left(v_{1}\right)\right)
$$

et

$$
B=D_{1} D_{2} J_{r}\left(v_{1}, \alpha_{r}\left(v_{1}\right)\right) \text {. }
$$

Ces opérateurs vérifient les hypothèses du lemme 4.1 ce qui permet d'en déduire que, pour tout $v_{1}$ de $\check{K}_{1}, D\left(\Phi_{r} \circ \alpha_{r}\right)\left(v_{1}\right)$ est un opérateur symétrique négatif de $\mathscr{L}\left(V_{1}, V_{1}\right)$. On a alors, pour tout couple $\left(v_{1}, w_{1}\right)$ de $K_{1}^{2}$ :

$$
\left(\left(\Phi_{r} \circ \alpha_{r}\right)\left(v_{1}\right)-\left(\Phi_{r} \circ \alpha_{r}\right)\left(w_{1}\right), v_{1}-w_{1}\right)_{1}=\left(D\left(\Phi_{r} \circ \alpha_{r}\right)\left(t_{1}\right) . \Psi_{1}, \Psi_{1}\right),
$$

avec $t_{1} \in \breve{K}_{1}$ et $\Psi_{1}=v_{1}-w_{1} \in V_{1}$ d'où le résultat énoncé.

PROPOSITION 4.3 : L'application $\Phi_{r} \circ \alpha_{r}$ est lipchitzienne de rapport $k$ sur $\stackrel{K}{\circ}_{1}$ ou encore

$$
\left\|\left(\Phi_{r} \circ \alpha_{r}\right)\left(v_{1}\right)-\left(\Phi_{r} \circ \alpha_{r}\right)\left(w_{1}\right)\right\|_{1} \leqq k\left\|v_{1}-w_{1}\right\|_{1}, \quad \forall\left(v_{1}, w_{1}\right) \in K_{1}^{2} .
$$


Démonstration : Il suffit d'utiliser (4.1), (4.4), (4.5), (4.6) et (2.4).

Remarque 4.1 : La démonstration des propositions $4.1,4.2$ et 4.3 reste valable dans le cas où l'hypothèse $(2.2)$ de forte convexe-concavité de la fonctionnelle $J$ est remplacée par l'hypothèse suivante :

$J$ est strictement convexe-concave sur $K_{1} \times K_{2}$,

$$
\begin{cases}\lim _{\substack{\left\|v_{1}\right\| \rightarrow+\infty \\ v_{1} \in K_{1}}} J\left(v_{1}, v_{2}\right)=+\infty, & \forall v_{2} \in K_{2}, \\ \lim _{\substack{\left\|v_{2}\right\| \rightarrow+\infty \\ v_{2} \in K_{2}}} J\left(v_{1}, v_{2}\right)=-\infty, & \forall v_{1} \in K_{1} .\end{cases}
$$

L'hypothèse de forte convexe-concavité (qui assure la convergence forte de la solution du problème $\left(P_{r}\right)$ vers la solution du problème $\left.(P)\right]$ nous permet de démontrer de plus que l'application $\left(-\Phi_{r} \circ \alpha_{r}\right)$ est non seulement monotone mais également fortement monotone, c'est-à-dire qu'il existe une constante positive $\alpha$, appellée constante de coercivité, telle que, pour tout couple $\left(v_{1}\right.$, $\left.w_{1}\right) \in \check{K}_{1} \times \check{K}_{1}$ on ait

$$
\left.-\left(\Phi_{r} \circ \alpha_{r}\right)\left(v_{1}\right)-\left(\Phi_{r} \circ \alpha_{r}\right)\left(w_{1}\right), v_{1}-w_{1}\right)_{1} \geqq \alpha\left\|v_{1}-w_{1}\right\|_{1}^{2} .
$$

si on suppose de plus :

$$
\left\|v_{2}^{-1} \circ D_{1} D_{2} J\left(v_{1}, v_{2}\right) . t_{1}\right\|_{2}^{2} \geqq T\left\|t_{1}\right\|_{1}^{2}, \quad \forall t_{1} \in V_{1} .
$$

Proposition 4.4: L'application $\left(-\Phi_{r} \circ \alpha_{r}\right)$ est fortement monotone sur $K_{1}$ et a pour constante de coercivité $\alpha=4 T_{1} T_{2} T / M_{1}^{2} M_{2}^{2}, T_{i}$ étant défni par (2.2) et $M_{i}$ étant un majorant de $\left\|D_{i}^{2} J\left(V_{1}, V_{2}\right)\right\|$ pour $i=1,2$.

Démonstration : $J$ étant fortement convexe-concave sur $K_{1} \times K_{2}$ d'après [1] on a

$$
\begin{gathered}
\left\langle D_{1}^{2} J\left(v_{1}, v_{2}\right)\left(w_{1}-v_{1}\right),\left(w_{1}-v_{1}\right)\right\rangle_{1} \geqq 2 T_{1}\left\|w_{1}-v_{1}\right\|_{1}^{2}, \\
\forall\left(v_{1}, v_{2}\right) \in K_{1} \times K_{2}, \quad \forall w_{1} \in K_{1}, \\
-\left\langle D_{2}^{2} J\left(v_{1}, v_{2}\right)\left(w_{2}-v_{2}\right), w_{2}-v_{2}\right\rangle_{2} \geqq 2 T_{2}\left\|w_{2}-v_{2}\right\|_{2}^{2} \\
\forall\left(v_{1}, v_{2}\right) \in K_{1} \times K_{2}, \quad \forall w_{2} \in K_{2} .
\end{gathered}
$$

Pour tout $\left(v_{1}, v_{2}\right) \in \dot{K}_{1} \times \dot{K}_{2}$ on montre alors que

$$
\begin{array}{cc}
\left\langle D_{1}^{2} J\left(v_{1}, v_{2}\right) \cdot t_{1}, t_{1}\right\rangle_{1} \geqq 2 T_{1}\left\|t_{1}\right\|_{1}^{2}, \quad \forall t_{1} \in V_{1}, \\
-\left\langle D_{2}^{2} J\left(v_{1}, v_{2}\right) \cdot t_{2}, t_{2}\right\rangle_{2} \geqq 2 T_{2}\left\|t_{2}\right\|_{2}^{2}, \quad \forall t_{2} \in V_{2} .
\end{array}
$$

On peut en déduire que. pour tout couple $\left(v_{1}, v_{2}\right) \in K_{1}^{\circ} \times K_{2}^{\circ}$, l'opérateur $\left[D_{1}^{2} J\left(v_{1}, v_{2}\right)\right]^{-1} \circ U_{1}$ de $\mathscr{L}\left(V_{1}, V_{1}\right)$ est coercif de constante de coercivité $2 T_{1} / M_{1}^{2}$, c'est-à-dire :

$$
\left(\left(\left[D_{1}^{2} J\left(v_{1}, v_{2}\right)\right]^{-1} \circ U_{1}\right) \cdot t_{1}, t_{1}\right)_{1} \geqq 2 T_{1}\left\|t_{1}\right\|_{1}^{2} / M_{1}^{2}, \quad \forall t_{1} \in V_{1} .
$$


De même l'opérateur

$$
\left[-C_{1}^{-1}\right] \circ\left[D_{2} D_{1} J\left(v_{1}, v_{2}\right)\right] \circ\left[D_{2}^{2} J\left(v_{1}, v_{2}\right)\right]^{-1} \circ\left[D_{1} D_{2} J\left(v_{1}, v_{2}\right)\right]
$$

de $2{ }^{\prime}\left(I, V_{1}\right)$ est coercif de constante de coercivite $2 T_{2} T / M_{2}^{2}$.

Posons

$$
\begin{gathered}
{\left[D_{1}^{2} J\left(\left(\Phi_{r} \circ \alpha_{r}\right)\left(v_{1}\right), \alpha_{r}\left(v_{1}\right)\right)\right]^{-1} \circ U_{1}=C_{1}\left(v_{1}\right),} \\
{\left[-U_{1}^{-1}\right] \circ\left[D_{2} D_{1} J\left(\left(\Phi_{r} \circ \alpha_{r}\right)\left(v_{1}\right), \alpha_{r}\left(v_{1}\right)\right)\right]} \\
\circ\left[D_{2}^{2} J\left(v_{1}, \alpha_{r}\left(v_{1}\right)\right]^{-1} \circ\left[D_{1} D_{2} J\left(v_{1}, \alpha_{r}\left(v_{1}\right)\right)\right]=C_{2}\left(v_{1}\right)\right.
\end{gathered}
$$

et

$$
C\left(v_{1}\right)=C_{1}\left(v_{1}\right) \cdot C_{2}\left(v_{1}\right) .
$$

On vient de voir que les opérateurs $C_{1}\left(v_{1}\right)$ et $C_{2}\left(v_{1}\right)$ de $\mathscr{L}\left(V_{1}, V_{1}\right)$ sont coercifs pour tout $v_{1}$ de $\mathscr{K}_{1}$ et on sait que ces deux opérateurs commutent (démonstration de la proposition 4.2). Montrons maintenant que leur produit $C\left(v_{1}\right)$ est également coercif.

En effet l'opérateur $C_{2}\left(v_{1}\right)$ étant positif il existe un unique opérateur positif $B_{2}\left(v_{1}\right)$ de $\mathscr{L}\left(V_{1}, V_{1}\right)$ tel que $B_{2}^{2}\left(v_{1}\right)=C_{2}\left(v_{1}\right)[10]$. De plus cet opérateur commute avec tout opérateur commutant avec $C_{2}\left(v_{1}\right)$ donc en particulier avec $C_{1}\left(v_{1}\right)$. Par suite

$$
\begin{aligned}
\left(C_{1}\left(v_{1}\right) C_{2}\left(v_{1}\right) t_{1}, t_{1}\right)_{1} & =\left(C_{1}\left(v_{1}\right) B_{2}\left(v_{1}\right) B_{2}\left(v_{1}\right) t_{1}, t_{1}\right)_{1} \\
& =\left(B_{2}\left(v_{1}\right) C_{1}\left(v_{1}\right) B_{2}\left(v_{1}\right) t_{1}, t_{1}\right) \\
= & \left(C_{1}\left(v_{1}\right) B_{2}\left(v_{1}\right) t_{1}, B_{2}\left(v_{1}\right) t_{1}\right) \geqq 2 T_{1}\left\|B_{2}\left(v_{1}\right) t_{1}\right\|_{1}^{2} / M_{1}^{2} .
\end{aligned}
$$

Mais

$$
\left\|B_{2}\left(v_{1}\right) t_{1}\right\|_{1}^{2}=\left(B_{2}\left(v_{1}\right) t_{1}, B_{2}\left(v_{1}\right) t_{1}\right)=\left(C_{2}\left(v_{1}\right) t_{1}, t_{1}\right) \geqq 2 T_{2}\left\|t_{1}\right\|_{1}^{2} T / M_{2}^{2}
$$

ce qui nous permet d'en déduire que l'opérateur $C\left(v_{1}\right)$ est coercif de constante de coercivité $4 T_{1} T_{2} T / M_{1}^{2} M_{2}^{2}=\alpha$.

De plus d'après (4.2). (4.3) et (4.4) :

$$
-\left(D\left(\Phi_{r} \circ \alpha_{r}\right)\left(v_{1}\right) \cdot t_{1}, t_{1}\right) \geqq\left(C\left(v_{1}\right) t_{1}, t_{1}\right), \quad \forall v_{1} \in K_{1}^{\circ}, \quad \forall t_{1} \in V_{1},
$$

donc $D\left(\Phi_{r} \circ \alpha_{r}\right)\left(v_{1}\right)$ est un opérateur coercif de constante de coercivité $\alpha$,d'où le résultat énoncé.

Afin de rendre évident le théorème 4.1 démontrons d'abord le lemme suivant :

Lemme 4.2: Soit $K$ un convexe fermé d'un espace de Hilbert réel $V$ et $f$ une application univoque de $K^{\circ}$ dans $K$, lipchitzienne de rapport $k$, d'opposée $(-f)$ 
fortement monotone (de rapport de coercivité $\gamma$ ) et admettant $\bar{x}$ pour unique point

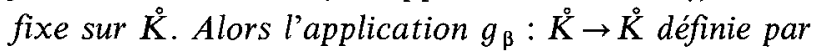

$$
g_{\beta}=(1-\beta) f+\beta I_{V}, \quad \beta \in \mathbb{R},
$$

admet $\bar{x}$ pour unique point fixe sur $\stackrel{K}{K}$, est contractante (c'est-à-dire lipchitzienne de rapport inférieur à 1) si le paramètre $\beta$ appartient à l'ensemble $I_{k, \gamma}$ défini par

$$
\left.I_{k, \gamma}=\right] \max \left(0, \frac{k^{2}-1}{k^{2}+2 \gamma+1}\right), 1[
$$

et a un rapport de contraction minimal si

$$
\beta=\beta_{\mathrm{opt}}=\frac{k^{2}+\gamma}{k^{2}+2 \gamma+1} .
$$

Démonstration: On vérifie aisément que l'élément $\bar{x}$ est un point fixe de $g_{\beta}$ et qu'il en est l'unique point fixe sur $\check{K}^{\circ}$.

Montrons maintenant que $g_{\beta}$ est lipchitzienne. On a

$$
\begin{aligned}
\left\|g_{\beta}(u)-g_{\beta}(v)\right\|_{V}^{2}=\beta^{2}\|u-v\|_{V}^{2}+(1-\beta)^{2}\|f(u)-f(v)\|_{V}^{2} & \\
& +2 \beta(1-\beta)(f(u)-f(v), u-v)_{V} .
\end{aligned}
$$

Pour $\beta \in] 0,1[$;

$$
2 \beta(1-\beta)(f(u)-f(v), u-v)_{V} \leqq-2 \beta(1-\beta) \gamma\|u-v\|_{V}^{2} .
$$

Par suite

$$
\left\|g_{\beta}(u)-g_{\beta}(v)\right\|_{V}^{2} \leqq\left(\beta^{2}\left(1+k^{2}+2 \gamma\right)-2 \beta\left(k^{2}+\gamma\right)+k^{2}\right)\|u-v\|_{V}^{2} .
$$

Posons

$$
[\theta(\beta)]^{2}=\beta^{2}\left(1+k^{2}+2 \gamma\right)-2 \beta\left(k^{2}+\gamma\right)+k^{2} .
$$

Si $\beta \in] 0,1\left[\right.$ l'application $g_{\beta}$ est donc lipchitzienne de rapport $\theta(\beta)$ et sera contractante si $\theta(\beta)<1$ ce qui est vérifié si $\beta$ appartient à l'intervalle $I_{k, \gamma}$ défini par (4.9).

De plus on peut montrer facilement que $\theta(\beta)$ est minimal pour

$$
\beta=\beta_{\mathrm{opt}}=\frac{k^{2}+\gamma}{k^{2}+2 \gamma+1}
$$

et dans ce cas

$$
[\theta(\beta)]^{2}=\frac{k^{2}-\gamma^{2}}{k^{2}+2 \gamma+1} .
$$

R.A I.R.O. Analyse numérıque/Numerıcal Analysıs 
THÉORÈME 4.1 : Soit $\beta$ appartenant $\grave{a} \mathbb{R}$. On considère l'application $T_{r, \beta}: \stackrel{\circ}{K}_{1} \rightarrow \dot{K}_{1}$ définie par

$$
T_{r, \beta}=(1-\beta)\left(\Phi_{r} \circ \alpha_{r}\right)+\beta I_{V}
$$

et on pose $\gamma=4 T_{1} T_{2} T / M_{1}^{2} M_{2}^{2}$.

L'application $T_{r, \beta}$ admet l'élément $\left(u_{1, r}\right)$ défini par (3.4) pour unique point fixe sur $\stackrel{K}{\mathrm{I}}_{\mathrm{f}}$ et est contractante quand $\beta$ appartient à $I_{k, \gamma}$ défini par (4.9). Le rapport de contraction $\theta(\beta)$ est alors minimal pour

$$
\beta=\beta_{\mathrm{opt}}=\frac{k^{2}+\gamma}{k^{2}+2 \gamma+1} \quad \text { et } \quad\left[\theta\left(\beta_{\mathrm{opt}}\right)\right]^{2}=\frac{k^{2}-\gamma^{2}}{k^{2}+2 \gamma+1} .
$$

Démonstration : L'application $\Phi_{r} \circ \alpha_{r}: \check{K}_{1} \rightarrow \check{K}_{1}$ vérifiant les hypothèses du lemme 4.2 il suffit d'appliquer ce lemme en prenant $f=\Phi_{r} \circ \alpha_{r}$.

Remarque 4.2 : A toute valeur de $\beta$ fixée correspond une infinité d'applications contractante $\left(T_{r, \beta}\right)_{r>0}$ de même rapport de contraction, minimum lorsque $\beta=\left(k^{2}+\gamma\right) /\left(k^{2}+2 \gamma+1\right)$.

\section{CONVERGENCE DE L'ALGORITHME}

Rappelons la méthode itérative présentée dans le paragraphe 3. Pour $i=1,2$ on considère une suite $r_{i, n}$ de nombres positifs décroissants vers zéro quand $n \rightarrow+\infty$. On pose $r_{n}=\left(r_{1, n}, r_{2, n}\right)$ et on note $\Phi_{n}$ l'application $\Phi_{r_{n}}, \alpha_{n}$ l'application $\alpha_{r_{n}}$ et $T_{n}$ l'application $T_{r_{n}, \beta_{0}}$ où $\beta_{0}$ est une valeur fixée du paramètre $\beta$ (on peut prendre par exemple $\left.\beta_{0}=\beta_{\text {opt }}\right)$. La suite $\left(x_{1, n}, x_{2, n}\right)$ est donc définie, à partir d'une valeur fixée $x_{1,0}$ de $K_{1}$, par

$$
\begin{gathered}
x_{2, n}=\alpha_{n}\left(x_{1, n-1}\right), \quad n \geqq 1, \\
x_{1, n}=T_{n}\left(x_{1, n-1}\right)=\left(1-\beta_{0}\right) \Phi_{n}\left(x_{2, n}\right)+\beta_{0} x_{1, n-1} .
\end{gathered}
$$

Proposition $5.1:$ La suite $\left(x_{1, n}, x_{2, n}\right)$ converge fortement vers $\left(u_{1}, u_{2}\right)$, solution du problème $(\mathrm{P})$, quand $n \rightarrow+\infty$.

Démonstration: Notons $u_{1, n}$ l'élément $u_{1, r_{n}}$, première composante du point de selle de $J_{r_{n}}$, dont on sait qu'il est l'unique point fixe de l'application $T_{n}$ sur $K_{1}$. L'application $T_{n}$ est contractante de rapport $\theta\left(\beta_{0}\right)$ donc

$$
\left\|u_{1, n}-x_{1, n}\right\|=\left\|T_{n}\left(u_{1, n}\right)-T_{n}\left(x_{1, n-1}\right)\right\| \leqq \theta\left(\beta_{0}\right)\left\|u_{1, n}-x_{1, n-1}\right\| .
$$

Mais

$$
\left\|u_{1, n}-x_{1, n-1}\right\| \leqq\left\|u_{1, n}-u_{1, n-1}\right\|+\left\|u_{1, n-1}-x_{1, n-1}\right\|,
$$


donc en posant

$$
e_{n}=\left\|u_{1, n}-u_{1, n-1}\right\| \quad \text { et } \quad f_{n}=\left\|u_{1, n}-x_{1, n}\right\| .
$$

on obtient :

et par suite

$$
f_{n} \leqq \theta\left(\beta_{0}\right) e_{n}+\theta\left(\beta_{0}\right) f_{n-1} \text {, }
$$

$$
0 \leqq f_{n} \leqq\left[\theta\left(\beta_{0}\right)\right]^{n} f_{0}+\sum_{k=1}^{n} e_{k}\left[\theta\left(\beta_{0}\right)\right]^{n+1-k}
$$

On sait que la suite $u_{1, n}$ converge fortement vers $u_{1}$ quand $n \rightarrow+\infty$ (paragraphe 3.1) donc la suite $e_{n} \rightarrow 0$ quand $n \rightarrow+\infty$. De plus $\left.\theta\left(\beta_{0}\right) \in\right] 0,1[$ donc en utilisant le lemme de Toeplitz [7] on en déduit que $f_{n}$ tend vers zéro quand $n \rightarrow+\infty$ et donc que $\left\|u_{1}-x_{1, n}\right\|$ tend lui aussi vers zéro quand $n++\infty$.

On vérifie alors aisément que $\left\|u_{2}-x_{2, n}\right\|$ tend vers zéro quand $n+\rightarrow \infty$.

Remarque 5.1 : On peut montrer qu'il y a également convergence forte du processus quand on effectue à chaque étape plusieurs itérations ce qui doit permettre dans certains cas d'améliorer la vitesse de convergence.

\section{APPROXIMATION NUMERIQUES : ITERATIONS APPROCHÉES}

Du point de vue pratique, appliquer l'algorithme ci-dessus conduit à la recherche, à chaque étape, d'un maximum et d'un minimum. Quelle que soit la méthode d'optimisation utilisée, elle ne donnera qu'une approximation de l'élément cherché aussi est-on conduit à étudier la convergence de la suite $\left(x_{1, n}^{*}\right.$, $x_{2, n}^{*}$ ) définie à partir de $x_{1,0}^{*}$ par

$$
\begin{array}{ll}
x_{2, n}^{*}=\alpha_{n}\left(x_{1, n-1}^{*}\right)+\eta_{n}, & n \geqq 1, \\
x_{1, n}^{*}=T_{n}\left(x_{1, n-1}^{*}\right)+\varepsilon_{n}, & n \geqq 1,
\end{array}
$$

où $\varepsilon_{n}$ et $\eta_{n}$ sont les erreurs commises en approchant respectivement $T_{n}\left(x_{1, n-1}^{*}\right)$ et $\alpha_{n}\left(x_{1, n-1}^{*}\right)$ à l'aide d'une méthode classique d'optimisation.

Proposition 6.1 : Si $\left\|\varepsilon_{n}\right\| \rightarrow 0$ et $\left\|\eta_{n}\right\| \rightarrow 0$ quand $n \rightarrow+\infty$ alors la suite $\left(x_{1, n}^{*}, x_{2, n}^{*}\right)$ définie par (6.1) et (6.2) converge fortement vers $\left(u_{1}, u_{2}\right)$ quand $n \rightarrow+\infty$.

Démonstration : Il suffit de montrer que $\left\|x_{1, n}^{*}-x_{1, n}\right\| \rightarrow 0$ quand $n++\infty$. On a

$$
\left\|x_{1, n}^{*}-x_{1, n}\right\| \leqq \theta\left(\beta_{0}\right)\left\|x_{1, n-1}^{*}-x_{1, n-1}\right\|+\left\|\varepsilon_{n}\right\| .
$$


En posant

$$
E_{n}=\left\|x_{1, n}^{*}-x_{1, n}\right\|
$$

on obtient :

$$
E_{n} \leqq \theta\left(\beta_{0}\right) E_{n-1}+\left\|\varepsilon_{n}\right\|
$$

et par suite

$$
E_{n} \leqq\left[\theta\left(\beta_{0}\right)\right]^{n} E_{0}+\sum_{k=1}^{n}\left[\theta\left(\beta_{0}\right)\right]^{n-k}\left\|\varepsilon_{k}\right\|
$$

En utilisant à nouveau le lemme de Toeplitz on en déduit le résultat énoncé.

\section{MAJORATION D'ERREUR}

Supposons que l'ensemble $K_{1}$ est borné. Dans le cas où l'ensemble $K_{2}$ et non l'ensemble $K_{1}$ est borné il suffit, de considérer l'algorithme défini par (3.9) et (3.10).

Posons

$$
a_{n}^{2}=\frac{1}{T_{1}}\left[\varepsilon_{1}\left(r_{1, n}\right) \sum_{1}^{q_{1}} \frac{1}{G_{1, j}\left(u_{1, n}\right)}+\varepsilon_{2}\left(r_{2, n}\right) \sum_{1}^{q_{2}} \frac{1}{G_{2, j}\left(u_{2, n}\right)}\right] .
$$

D'après $(3.6)$ on $\mathrm{a}$

$$
\left\|u_{1}-u_{1, n}\right\| \leqq a_{n} \quad \text { et } \quad e_{k}=\left\|u_{1, k}-u_{1, k-1}\right\| \leqq a_{k}+a_{k-1} .
$$

L'ensemble $K_{1}$ étant borné il existe une constante positive $M_{1}$ telle que

$$
\left\|v_{1}\right\| \leqq M_{1}, \quad \forall v_{1} \in K_{1} .
$$

Par suite

$$
\left\|u_{1,0}-x_{1,0}\right\| \leqq 2 M_{1} .
$$

De (5.3) on peut donc déduire :

$$
\left\|u_{1}-x_{1, n}\right\| \leqq 2 M_{1}\left[\theta\left(\beta_{0}\right)\right]^{n}+\sum_{k=1}^{n}\left(a_{k}+a_{k-1}\right)\left[\theta\left(\beta_{0}\right)\right]^{n+1-k}+a_{n} .
$$

Dans le cas des itérations approchées on obtient :

$$
\begin{aligned}
\left\|u_{1}-x_{1, n}^{*}\right\| \leqq 4 M_{1}\left[\theta\left(\beta_{0}\right)\right]^{n} & +a_{n} \\
& +\sum_{k=1}^{n} \theta\left(\beta_{0}\right)^{n-k}\left(\left\|\varepsilon_{k}\right\|+\theta\left(\beta_{0}\right)\left(a_{k}+a_{k-1}\right)\right) .
\end{aligned}
$$


Du point de vue numerique le second membre de $\left(\begin{array}{ll}7 & 2\end{array}\right)$ n'est pas connu rigoureusement en raison de la presence des elements $u_{1 k}$ dont on ne connaît qu'une approximation $x_{1}^{*}$

De même on etablit facilement des majorations de $\left\|u_{2}-x_{2}\right\|$ et de $\left\|u_{2}-x_{2}^{*}\right\|$

\section{BIBLIOGRAPHIE}

1 A Auslender, Problemes de minimax via lanalyse convexe et les inegalites variationnelles Theorle et algorithmes, $\mathrm{n}^{\circ} 77$, Lectures Notes in Economics and Mathematical Systems, Springer-Verlag, 1972

2 Y Cherruault, Une methode directe de minimisation et applications, R I R O, vol $10,1968, \mathrm{p} 31-52$

3 A V Fiacco, Comments on the paper of C Caroll, Operations Reseach, vol 9, $\mathrm{n}^{\circ} 2$, mars-avril 1961, p 184-185

4 A V Fiacco et G P McCormick, The Sequential Unconstrained Minimization Technique for Non linear Programming, a Primal dual Method, Managment Science, vol $10, \mathrm{n}^{\circ} 2,1964, \mathrm{p} 360-366$

5 A V Fiacco et G P McCormick, Non Linear Programming Sequential Unconstrained Minımizatıon Technique, Wiley, New York, 1970

$6 \mathrm{~J}$ Morgan-Sciarrino, Approximation interne de problemes de point de selle avec contraintes, Boll Un Mat Ital, (5), vol 15-B, no 1, 1978, p 147-160

7 J M ORTEgA et W C ReInboldt, Iterative Solutions of Non Linear Equations in Several Var rables, Academic Press, 1970

$8 \mathrm{H}$ SASAI, An Interior Penalty Method for Minimax Problems with Constraints, S I A M Control, vol 12, $\mathrm{n}^{\circ} 4,1974, \mathrm{p}$ 643-649

9 L Schu ART7 Cours d analis Hermann Paris 1967

10 A C LaAnev Linear Analists North-Holland Amsterdam 1956 